\title{
Management of a Preterm Infant with Renal Tubular Dysgenesis: A Case Report and Review of the Literature
}

\author{
Tomomi Kondoh, ${ }^{1}$ Yuri Kawai, ${ }^{1}$ Yuji Matsumoto, ${ }^{1}$ Naonori Kumagai, ${ }^{1}$ \\ Masafumi Miyata, ${ }^{1}$ Kazuki Tanaka, ${ }^{2}$ Satoshi Hibino, ${ }^{2}$ Naoya Fujita $^{2}$ and \\ Yohei Ikezumi ${ }^{1}$
}

${ }^{1}$ Department of Pediatrics, Fujita Health University School of Medicine, Toyoake, Aichi, Japan

${ }^{2}$ Department of Nephrology, Aichi Children's Health and Medical Center, Obu, Aichi, Japan

\begin{abstract}
Renal tubular dysgenesis (RTD) is the absence or poor development of the renal proximal tubules caused by gene mutations in the renin-angiotensin system. Although RTD has been considered fatal, improving neonatal intensive care management has enhanced survival outcomes. However, little has been reported on the survival of extremely preterm infants. This study reports the survival of an extremely preterm infant with RTD and discusses the appropriate management of RTD by reviewing the literature. A female infant weighing $953 \mathrm{~g}$ was delivered at 27 weeks' gestation by Cesarean section because of oligohydramnios. She exhibited severe persistent pulmonary hypertension, severe systemic hypotension, and renal dysfunction shortly after birth. Respiratory management was successfully undertaken using nitric oxide inhalation and high-frequency oscillatory ventilation. Desmopressin was effective in maintaining her blood pressure and urinary output. She was diagnosed with RTD based on genetic testing, which revealed a compound heterozygous mutation in the angiotensin-converting enzyme gene in exon 18 (c.2689delC; p.Pro897fs) and exon 20 (c.3095dupT; p.Leu1032fs). At 2 years, she started receiving oral fludrocortisone for treating persistently high serum creatinine levels, which was attributed to nephrogenic diabetes insipidus caused by RTD. Subsequently, her urine output decreased, and renal function was successfully maintained. Currently, there is no established treatment for RTD. Considering cases reported to date, treatment with vasopressin and fludrocortisone appears to be most effective for survival and maintenance of renal function in patients with RTD. This study presents the successful management of RTD using this strategy in an extremely preterm infant.
\end{abstract}

Keywords: angiotensin-converting enzyme; fludrocortisone; renal tubular dysgenesis; renin-angiotensin system; vasopressin

Tohoku J. Exp. Med., 2020 September, 252 (1), 9-14.

\section{Introduction}

Autosomal recessive renal tubular dysgenesis (RTD) is a severe disorder of renal tubular development resulting in the absence or poor development of the renal proximal tubules (Allanson et al. 1983). The disorder is characterized by an early onset of persistent fetal anuria leading to oligohydramnios and Potter sequence (pulmonary hypoplasia, abnormal facies and limb abnormalities), as well as skull ossification. In most cases, early death occurs due to anuria, pulmonary hypoplasia, and refractory arterial hypotension. The disease is associated with mutations in the genes encoding several components of the renin-angiotensin system (RAS), including angiotensinogen (AGT), renin (REN), angiotensin-converting enzyme (ACE), and angiotensin II receptor type 1 (AGTR1) (Gribouval et al. 2012). Any gene mutation of encoding RAS causes the lack of angiotensin II (ANG II) activity. Since ANG II plays a critical role in nephrovascular development, inhibition of ANG II during the perinatal period results in the kidney with profound structural abnormalities; most prominent are hypertrophy of arterial vasculatures and atrophy of the papilla. The hypertrophy of arterial vasculatures consequently causes the absence or severe atrophy of the tubules and

Received May 26, 2020; revised and accepted July 31, 2020. Published online August 19, 2020; doi: 10.1620/tjem.252.9.

Correspondence: Tomomi Kondoh, Department of Pediatrics, Fujita Health University School of Medicine, 1-98 Dengakugakubo, Kutsukake-cho, Toyoake, Aichi 470-1192, Japan.

e-mail: tomomi-h@ fujita-hu.ac.jp

(C)2020 Tohoku University Medical Press. This is an open-access article distributed under the terms of the Creative Commons Attribution-NonCommercial-NoDerivatives 4.0 International License (CC-BY-NC-ND 4.0). Anyone may download, reuse, copy, reprint, or distribute the article without modifications or adaptations for non-profit purposes if they cite the original authors and source properly.

https://creativecommons.org/licenses/by-nc-nd/4.0/ 
reduced glomerular size and number (Matsusaka et al. 2002). Secondary RTD has been observed; however, it is distinct from hereditary RTD. Secondary RTD occurs in various situations which lead to hypoperfusion of the kidney (e.g., the donor twin of severe twin-twin transfusion syndrome, fetuses affected with congenital haemochromatosis, and fetuses exposed to RAS blockers) (Gubler 2014). Because the management of arterial hypotension and anuria influences the survival of an infant with RTD, early diagnosis of an anuric fetus with insufficient concrete findings on a fetal renal ultrasound is critical. Although RTD has typically been known as a fatal disease, several cases of survival after the neonatal period have recently been reported (Uematsu et al. 2006, 2009; Zingg-Schenk et al. 2008; Danilov et al. 2010; Schreiber et al. 2010; Kim et al. 2012; Hibino et al. 2015; Richer et al. 2015; Ruf et al. 2018). We report here a case of an extremely preterm infant (among preterm infants, those born less than 28 weeks of gestation) with RTD managed without renal replacement therapy and discuss the most appropriate management of RTD based on the course of treatment (for this case) and a review of the literature.

\section{Case Presentation}

A female infant was born to a 35-year-old mother who was pregnant for the second time. There was no obvious family history, and the mother did not take any medications during her pregnancy. The infant's 2-year-old brother was healthy. Oligohydramnios was observed at 24 weeks of pregnancy, at which time the mother was admitted to the Department of Obstetrics at our hospital. There was no amniotic fluid present, and ultrasonography revealed a normal fetal urinary tract and kidneys. Amnioinfusion was attempted; however, it was unsuccessful because there was no adequate space to safely puncture the amniotic cavity. Because of persistent oligohydramnios, the infant was born at the 27th week of gestation by Cesarean section. Her birth weight was $953 \mathrm{~g}$, which was appropriate for the gestational age. Apgar score was 8 at 1 and 5 minutes, respectively. Since severe respiratory failure caused by pulmonary hypoplasia due to Potter sequence was predicted, inhaled nitric oxide therapy (iNO) was initiated during resuscitation. Similarly, she was immediately intubated and administered artificial surfactant. She developed pulmonary hypoplasia, and respiratory management was successfully undertaken using high-frequency oscillatory ventilation (HFOV) and continued iNO therapy. During the first day of life, she presented with oliguria and severe hypotension (mean blood pressure [BP] $15 \mathrm{mmHg}$ ), which responded poorly to rapid volume loading, catecholamine, and hydrocortisone. Desmopressin therapy $(0.001 \mathrm{IE} / \mathrm{kg} /$ min) was initiated on day 5, resulting in blood pressure elevation and gradual improvement in kidney function. On day 10 , iNO was stopped, and she was extubated on day 30 .

Laboratory testing at 7 months revealed asymptomatic renal failure and renal tubular acidosis (serum creatinine
[sCr] $0.46 \mathrm{mg} / \mathrm{dL}$, blood urea nitrogen $16.6 \mathrm{mg} / \mathrm{dL}$, cystatin C $2.0 \mathrm{mg} / \mathrm{dL}, \mathrm{Na} 143 \mathrm{mEq} / \mathrm{L}, \mathrm{K} 5.8 \mathrm{mEq} / \mathrm{L}, \mathrm{Cl} 118 \mathrm{mEq} / \mathrm{L}$, $\mathrm{pH}$ 7.31, $\mathrm{PCO}_{2} 28.5 \mathrm{mmHg}, \mathrm{HCO}_{3} 14.2 \mathrm{mEq} / \mathrm{L}$, base excess $-10.8 \mathrm{mEq} / \mathrm{L}$ ). Sodium hydrogen carbonate and an antihyperkalemic agent were administered to control electrolytes and bicarbonate. Additional investigation showed high renin activity: $23 \mathrm{ng} / \mathrm{mL} / \mathrm{h}$ (normal range: $0.2-2.7 \mathrm{ng} / \mathrm{mL} / \mathrm{h}$ ), high active renin concentration: $9,300 \mathrm{pg} / \mathrm{mL}$ (normal range: $3.2-36 \mathrm{pg} / \mathrm{mL}$ ), and normal aldosterone: $52.1 \mathrm{pg} / \mathrm{mL}$ (normal range: $35-240 \mathrm{pg} / \mathrm{mL}$ ).

In the diagnosis, RTD was suspected based on the clinical findings (oligohydramnios, severe hypotension, renal dysfunction with insufficient concrete findings on renal ultrasound, and hypoaldosteronism) and was confirmed by gene analysis. Testing revealed a novel compound heterozygous mutation: $A C E$ (NM_000789); exon18: c.2689delC (p.Pro897fs) and exon20: c.3095dupT (p. Leu1032fs). The c.2689delC was observed in the mother, whereas c.3095dupT was observed in the father. The mutations were validated by Sanger sequencing (Fig. 1.).

At 14 months old, she underwent percutaneous gastrostomy, which was performed to aid her oral intake. Subsequently, her sCr level elevated to a range of 1.2 and $1.8 \mathrm{mg} / \mathrm{dL}$ (reference value for her height is approximately $0.2 \mathrm{mg} / \mathrm{dL}$ ), and hydration treatment of at least $2 \mathrm{~L} /$ day was initiated. Consequently, her sCr level was decreased and was maintained below $1.0 \mathrm{mg} / \mathrm{dL}$. Blood tests equally revealed hyperkalemia, and treatment with polystyrene sulfonate, an antihyperkalemic agent, was initiated. Supposedly, these findings were due to dehydration and renal ischemia caused by nephrogenic diabetes insipidus associated with RTD. Despite dehydration, her plasma aldosterone levels did not increase, suggesting that renal diabetes insipidus was caused by insufficient aldosterone action. Therefore, at age 2 years, she started receiving oral fludrocortisone based on the presence of persistent conditions. After starting fludrocortisone, hyperkalemia improved and $\mathrm{sCr}$ levels were maintained at $<0.5 \mathrm{mg} / \mathrm{dL}$ (estimated glomerular filtration rate [eGFR]: $60-70 \mathrm{~mL} /$ $\min / 1.73 \mathrm{~m}^{2}$ ). Her potassium level remained normal, even after the hydration and treatment with the antihyperkalemic agent were significantly reduced.

The patient is presently 4 years old with chronic kidney disease stage 2 (eGFR: $\left.73 \mathrm{~mL} / \mathrm{min} / 1.73 \mathrm{~m}^{2}\right)$. She is currently being treated with fludrocortisone $(0.08 \mathrm{mg} /$ day $)$ and continues to undergo hydration and treatment with sodium bicarbonate, antihyperkalemic agent, and vitamin D to prevent severe tubular disorders. Her cognitive and psychomotor functions are gradually improving, and conversation and walking are presently possible.

Informed consent was obtained from the mother for publication of this case.

\section{Discussion}

This study reports the survival and successful management of an extremely preterm infant diagnosed with RTD. 


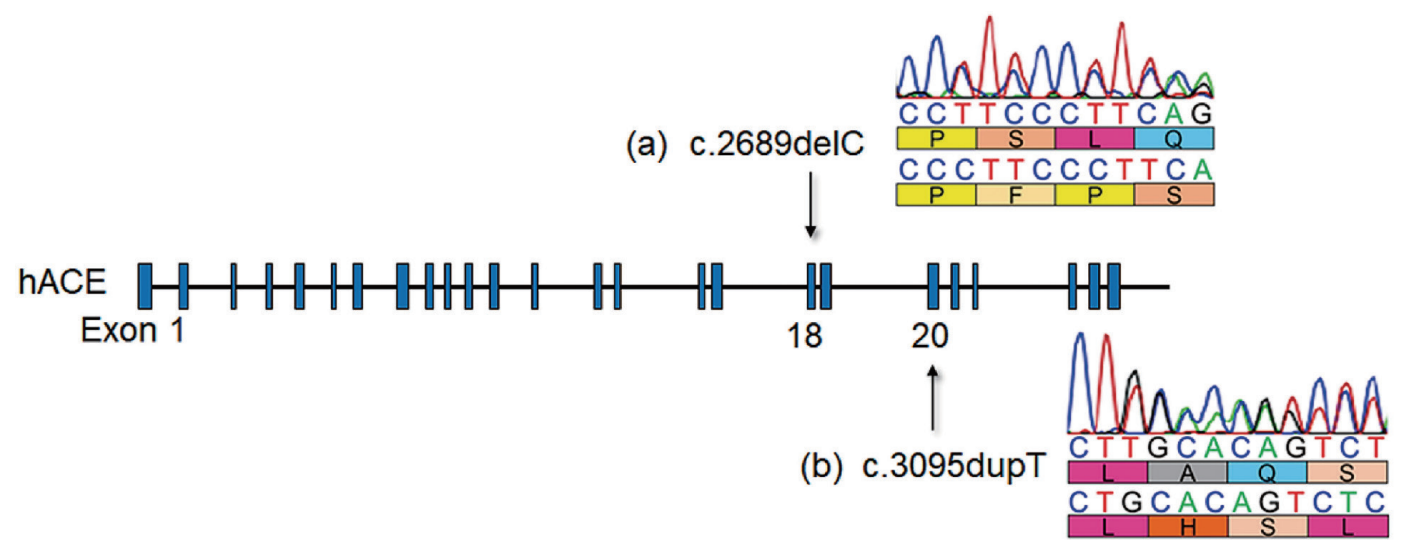

Fig. 1. Schematic representation of angiotensin-converting enzyme gene structure.

The boxes denote exons. The compound heterozygous mutations identified are indicated by arrows. The upper base sequence is that of the patient, whereas the lower base sequence is the control in Sanger sequencing. (a) Sequencing of exon 18 reveals a heterozygous $\mathrm{C}$ deletion at position 2689. (b) Sequencing of exon 20 shows a heterozygous $\mathrm{T}$ duplication at position 3095 .

According to the literature search, approximately 150 cases of RTD have been reported so far; however, only 13 of the individuals, including the child in this case, are known to have survived as of this report (Uematsu et al. 2006, 2009; Zingg-Schenk et al. 2008; Danilov et al. 2010; Schreiber et al. 2010; Gribouval et al. 2012; Kim et al. 2012; Hibino et al. 2015; Richer et al. 2015; Ruf et al. 2018). (We conducted a search of the PubMed databases through April 2020 using the keyword "renal tubular dysgenesis." All reports associated with RTD were identified.) Among all cases found, only the reports of long-term survivors whose detailed clinical course is provided and the gene mutation identified, are summarized in Table 1. The neonate in this report has the earliest preterm birth and the lowest birthweight of all reported cases.

The locus of human $A C E$ gene is $17 \mathrm{q} 23.3$ on chromosome 17; this gene consists of 26 exons (Mattei et al. 1989). The $A C E$ gene encodes ACE that is expressed mainly in the lung and in other tissues, including renal vascular endothelial cells and renal epithelial cells (Harmer et al. 2002) and catalyzes the conversion of angiotensin I to a physiologically active peptide, ANG II. ANG II regulates the blood pressure and electrolyte balance. As described above, the compound heterozygous mutations detected in the patient are both frameshift mutations. Presumably, the mutations impair the catalytic function of ACE; hence, mutations in $A C E$ observed in this patient were considered the causative gene mutations which would consequently cause loss of ANG II function.

For newborns with RTD, the most important factor in long-term survival is neonatal management, and the first step in managing RTD is early diagnosis. Although diagnosing RTD may be difficult, characteristic clinical features can aid the clinician in their determinations. Most RTD cases are characterized by oligohydramnios, which may be recognized at 20 weeks of pregnancy, sometimes earlier, and which persists throughout pregnancy. As in this case, the most important characteristic clue is oligohydramnios observed during the early stages of pregnancy, accompanied by fetal ultrasound showing an absence of major kidney or urinary tract abnormalities. Fetal anuria often leads to oligohydramnios and Potter sequence (clubbed feet, pulmonary hypoplasia, and cranial anomalies). Secondary RTD is associated with intrauterine growth retardation, whereas fetuses with hereditary RTD often exhibit normal development (Gubler 2014). Most often, the patient may die in utero or soon after birth due to respiratory failure, persistent anuria, and severe low BP resistant to the usual vasopressor therapies (rapid volume loading, catecholamines, and glucocorticoids). Thus, the first step to rescuing RTD neonates is to prevent the onset of Potter sequence or to minimize its effects. Managing extremely preterm infants is often difficult, even in the absence of underlying disease; however, it is even more challenging to manage extremely preterm RTD infants with Potter sequence. Therefore, an effective strategy may be to perform a Cesarean section before Potter sequence due to the progression of oligohydramnios and an increase in the severity of pulmonary hypoplasia. Since pulmonary hypertension due to pulmonary hypoplasia was expected in this patient, iNO was used (during resuscitation) shortly after birth. In fact, right to left circulatory shunts were observed immediately after birth, which indicated the presence of pulmonary hypertension. Severe respiratory failure was successfully managed with HFOV and continued iNO therapy.

The second step in the rescue of an RTD newborn is the treatment of refractory hypotension. Analysis of previous reports has shown that vasopressin has been used for the treatment of hypotension in 3 of $10(30 \%)$ long-term survivors. Since the central pathology of RTD is caused by mutation of genes involved in the RAS, which regulates blood pressure and the balance of fluids and salts in the body, defects in angiotensin II (AT II) result in severe hypotension. AT II not only acts as a vasoconstrictor but also 
T. Kondoh et al.

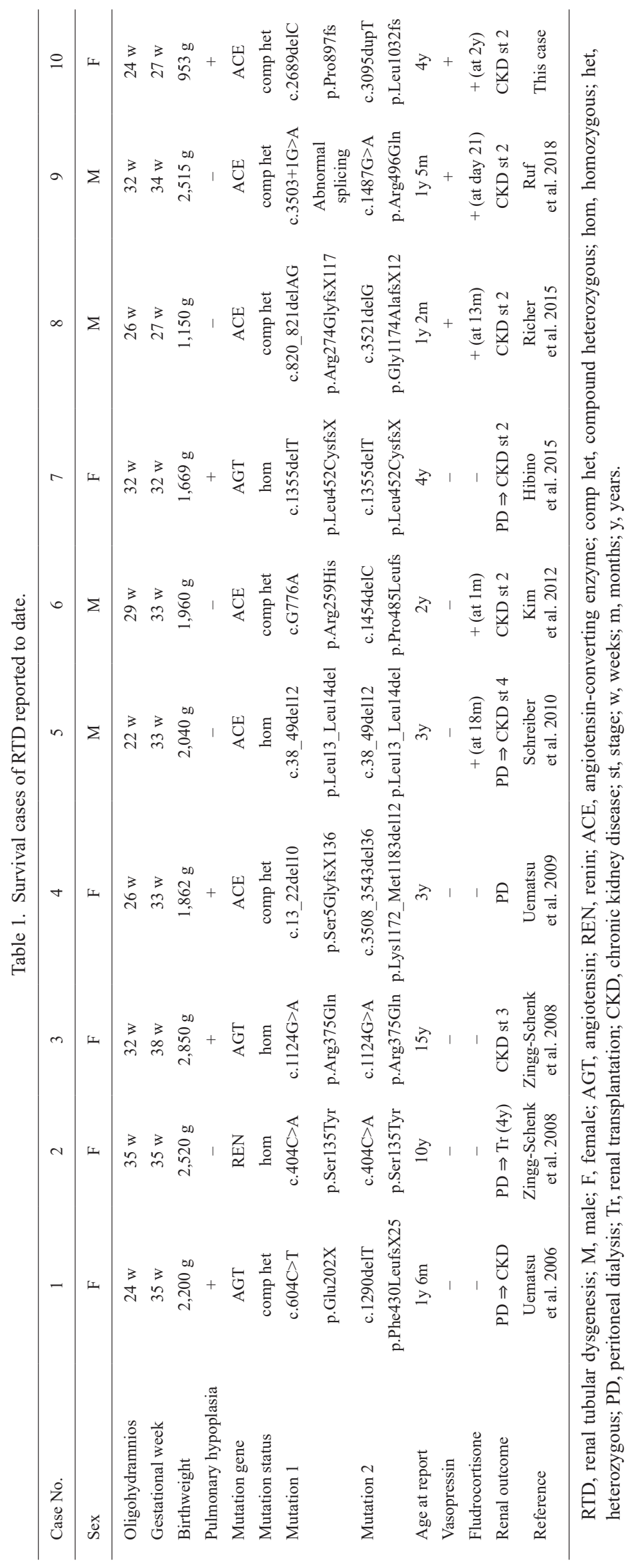


stimulates the adrenal gland and the posterior pituitary gland to release aldosterone and vasopressin, respectively. These hormones increase the BP by promoting the reabsorption of water from the renal distal tubules and by reducing urinary excretion from the collecting ducts (Bernstein et al. 2013). In the neonatal stage of development, production of vasopressin exceeds that of aldosterone due to immature tubular function (Bourchier 2005). Therefore, treatment with vasopressin could compensate for the lack of pressor effect of AT II and aldosterone in RTD newborns. However, as renal function develops with growth, the lack of aldosterone action becomes apparent, as with the patient in this case.

The third step in managing an RTD newborn is the maintenance of renal function. After the infant in this case recovered from neonatal crisis, the primary clinical manifestations of the disorder were the result of defects in the RAS. Because of this, it was critical to initiate treatment for hypoaldosteronism, as it can lead to polyuria, which can consequently cause dehydration, renal failure due to ischemia, and hyperkalemia. Fludrocortisone was administered, and indeed, renal function and hyperkalemia were significantly improved. This result is supported by the literature, as $50 \%$ of the reported RTD survivors were administered fludrocortisone, in all of whom normal renal function was maintained without renal replacement therapy (Table 1). Some RTD survivors, including this patient, were administered hydrocortisone during treatment. While hydrocortisone can elicit both glucocorticoid and mineralocorticoid effects on hypotension, it had no effect in this case. Since hydrocortisone has only $1 / 125$ of the mineralocorticoid action compared with fludrocortisone at the same dose, the usual dose of hydrocortisone used in newborns may not be sufficient enough to reverse hypotension in RTD neonates. Alternatively, neonates may respond poorly to aldosterone due to immature renal tubular function and are at risk for hyponatremia without proper sodium supplementation, even more refractory, especially in preterm infants (Bourchier 2005). Although sCr levels started elevating in this patient at 14 months old and treatment with fludrocortisone was commenced at 2 years old, there is a report of successful renal function maintenance in a patient who underwent fludrocortisone treatment, which was commenced 21 days after birth (Ruf et al. 2018). Therefore, it is difficult to determine when to start fludrocortisone; however, administration of fludrocortisone appears to be useful, regardless of the timing.

Gribouval et al. (2012) reviewed a series of 54 distinct mutations identified in 48 unrelated families and reported that the severity of the clinical course was similar for all individuals, regardless of the location of the mutation. Recently, Fila et al. (2020) reported 3 patients with RTD due to biallelic mutations in the RAS who presented with atypically late chronic renal failure. These results suggest it may be difficult to predict the severity of the disease simply by identifying the genetic mutation. Thus, the RTD man- agement course presented here may not always be appropriate for patients with RAS mutations; however, it may be useful for patients with typical RTD clinical manifestations.

Due to the limited number of patients who survive the neonatal period, clinical data may not be sufficient to establish management of RTDs. We believe our presented management is currently the best way to manage RTD newborns. With the development of neonatal care and/or genetic diagnostics, early diagnosis of RTDs and new treatments will be available in the future.

In conclusion, this study presents a report on the survival and successful management of an extremely preterm infant diagnosed with RTD. The results suggest that, when oligohydramnios manifests in the second trimester without fetal kidney and urinary tract anomalies, the possibility of RTD should be considered. Additionally, Cesarean section should be performed before pulmonary hypoplasia becomes more severe. Similarly, proper respiratory management using iNO should be performed immediately after birth. Finally, we recommend using vasopressin for the treatment of neonatal hypotension, followed by the administration of fludrocortisone at an early stage of the clinical course.

\section{Acknowledgments}

We would like to thank Hiroki Kurahashi (Division of Molecular Genetics, Institute for Comprehensive Medical Science, Fujita Health University) for the gene analysis.

\section{Conflict of Interest}

The authors declare no conflict of interest.

\section{References}

Allanson, J.E., Pantzar, J.T. \& MacLeod, P.M. (1983) Possible new autosomal recessive syndrome with unusual renal histopathological changes. Am. J. Med. Genet., 16, 57-60.

Bernstein, K.E., Ong, F.S., Blackwell, W.L., Shah, K.H., Giani, J.F., Gonzalez-Villalobos, R.A., Shen, X.Z., Fuchs, S. \& Touyz, R.M. (2013) A modern understanding of the traditional and nontraditional biological functions of angiotensinconverting enzyme. Pharmacol. Rev., 65, 1-46.

Bourchier, D. (2005) Plasma aldosterone levels in the 1st week of life in infants of less than 30 weeks gestation. Eur. J. Pediatr., 164, 141-145.

Danilov, S.M., Kalinin, S., Chen, Z., Vinokour, E.I., Nesterovitch, A.B., Schwartz, D.E., Gribouval, O., Gubler, M.C. \& Minshall, R.D. (2010) Angiotensin I-converting enzyme Gln1069Arg mutation impairs trafficking to the cell surface resulting in selective denaturation of the C-domain. PLoS One, 5, e10438.

Fila, M., Morinière, V., Eckart, P., Terzic, J., Gubler, M.C., Antignac, C. \& Heidet, L. (2020) Bi-allelic mutations in reninangiotensin system genes, associated with renal tubular dysgenesis, can also present as a progressive chronic kidney disease. Pediatr. Nephrol., 35, 1125-1128.

Gribouval, O., Morinière, V., Pawtowski, A., Arrondel, C., Sallinen, S.L., Saloranta, C., Clericuzio, C., Viot, G., Tantau, J., Blesson, S., Cloarec, S., Machet, M.C., Chitayat, D., Thauvin, C., Laurent, N., et al. (2012) Spectrum of mutations in the renin-angiotensin system genes in autosomal recessive renal tubular dysgenesis. Hum. Mutat., 33, 316-326.

Gubler, M.C. (2014) Renal tubular dysgenesis. Pediatr. Nephrol., 
29, 51-59.

Harmer, D., Gilbert, M., Borman, R. \& Clark, K.L. (2002) Quantitative mRNA expression profiling of ACE 2, a novel homologue of angiotensin converting enzyme. FEBS Lett., 532, 107-110.

Hibino, S., Sasaki, H., Abe, Y., Hojo, A., Uematsu, M., Sekine, T. \& Itabashi, K. (2015) Renal function in angiotensinogen gene-mutated renal tubular dysgenesis with glomerular cysts. Pediatr. Nephrol., 30, 357-360.

Kim, S.Y., Kang, H.G., Kim, E.K., Choi, J.H., Choi, Y. \& Cheong, H.I. (2012) Survival over 2 years of autosomal-recessive renal tubular dysgenesis. Clin. Kidney J., 5, 56-58.

Matsusaka, T., Miyazaki, Y. \& Ichikawa, I. (2002) The renin angiotensin system and kidney development. Annu. Rev. Physiol., 64, 551-561.

Mattei, M.G., Hubert, C., Alhenc-Gelas, F., Roeckel, N., Corvol, P. \& Soubrier, F. (1989) Angiotensin-I converting enzyme gene is on chromosome-17. Cytogenet. Cell. Genet., 51, 10411045.

Richer, J., Daoud, H., Geier, P., Jarinova, O., Carson, N., Feberova, J., Ben Fadel, N., Unrau, J., Bareke, E., Khatchadourian, K., Bulman, D.E., Majewski, J., Boycott, K.M. \& Dyment, D.A. (2015) Resolution of refractory hypotension and anuria in a premature newborn with loss-of-function of ACE. Am. J. Med. Genet. A, 167, 1654-1658.

Ruf, K., Wirbelauer, J., Beissert, A. \& Frieauff, E. (2018) Successful treatment of severe arterial hypotension and anuria in a preterm infant with renal tubular dysgenesis: a case report. Matern. Health Neonatol. Perinatol., 4, 27.

Schreiber, R., Gubler, M.C., Gribouval, O., Shalev, H. \& Landau, D. (2010) Inherited renal tubular dysgenesis may not be universally fatal. Pediatr. Nephrol., 25, 2531-2534.

Uematsu, M., Sakamoto, O., Nishio, T., Ohura, T., Matsuda, T., Inagaki, T., Abe, T., Okamura, K., Kondo, Y. \& Tsuchiya, S. (2006) A case surviving for over a year of renal tubular dysgenesis with compound heterozygous angiotensinogen gene mutations. Am. J. Med. Genet. A, 140, 2355-2360.

Uematsu, M., Sakamoto, O., Ohura, T., Shimizu, N., Satomura, K. \& Tsuchiya, S. (2009) A further case of renal tubular dysgenesis surviving the neonatal period. Eur. J. Pediatr., 168, 207-209.

Zingg-Schenk, A., Bacchetta, J., Corvol, P., Michaud, A., Stallmach, T., Cochat, P., Gribouval, O., Gubler, M.C. \& Neuhaus, T.J. (2008) Inherited renal tubular dysgenesis: the first patients surviving the neonatal period. Eur. J. Pediatr., 167, 311-316. 